Innovations in Incidence Geometry

Volume 15 (2017), Pages 229-264

ISSN 1781-6475

\title{
Counting points and acquiring flesh
}

\author{
Koen Thas*
}

To my father, $70+$ and still going strong.

\begin{abstract}
This set of notes is based on a lecture I gave at " 50 years of Finite Geometry - A conference on the occasion of Jef Thas's 70th birthday," in November 2014. It consists essentially of three parts: in a first part, I introduce some ideas which are based in the combinatorial theory underlying $\mathbb{F}_{1}$, the field with one element. In a second part, I describe, in a nutshell, the fundamental scheme theory over $\mathbb{F}_{1}$ which was designed by Deitmar. The last part focuses on zeta functions of Deitmar schemes, and also presents more recent work done in this area.
\end{abstract}

Keywords: Field with one element, Deitmar scheme, loose graph, zeta function, Weyl geometry

MSC 2010: 11G25, 11D40, 14A15, 14G15

\section{Contents}

1 Introduction $\quad 230$

2 Combinatorial theory $\quad 239$

3 Deninger-Manin theory 243

4 Deitmar schemes $\quad 245$

*I want to thank two anonymous referees for various detailed remarks and suggestions on an earlier draft of this paper. I also want to thank Andries Brouwer for a highly helpful communication on graphs that satisfy the Buekenhout-Shult "one-or-all axiom." Finally, a final version of this paper was produced while I was visiting Matilde Marcolli at Caltech in April 2017, whose hospitality is gratefully acknowledged. 
6 Kurokawa theory 250

7 Graphs and zeta functions $\quad 253$

A Acquiring flesh (2) - The Weyl functor depicted 261

\section{Introduction}

For a class of incidence geometries which are defined (for instance coordinatized) over fields, it often makes sense to consider the "limit" of these geometries when the number of field elements tends to 1 . As such, one ends up with a guise of a "field with one element, $\mathbb{F}_{1}$ " through taking limits of geometries. A general reference for $\mathbb{F}_{1}$ is the recent monograph [23].

\subsection{Example: projective planes}

For instance, let the class of geometries be the classical projective planes $\mathbf{P G}(2, k)$ defined over commutative fields $k$. Then the number of points per line and the number of lines per point of such a plane is

$$
|k|+1
$$

so in the limit, the "limit object" should have $1+1$ points incident with every line. On the other hand, we want that the limit object remains an axiomatic projective plane, so we still want it to have the following properties:

(i) any two distinct lines meet in precisely one point;

(ii) any two distinct points are incident with precisely one line (the dual of (i));

(iii) not all points are on one and the same line (to avoid degeneracy).

It is clear that such a limit projective plane ("defined over $\mathbb{F}_{1}$ ") should be an ordinary triangle (as a graph).

\subsection{Example: generalized polygons}

Projective planes are, by definition, generalized 3-gons. Generalizing the situation to generalized $n$-gons, $n \geq 3$, a limit generalized $n$-gon becomes an 
ordinary $n$-gon (as a graph). The easiest way to see this is through the polygonal definition of generalized $n$-gons: if $\mathcal{E}$ is the union of the set of points and the set of lines (which are assumed to be disjoint without loss of generality), then one demands that:

PD1 there are no sub $m$-gons with $2 \leq m \leq n-1$;

PD2 any two elements of $\mathcal{E}$ are inside at least one $n$-gon, and

PD3 there exist $(n+1)$-gons.

There is a constant $c \neq 0,1$ such that any line is incident with $c+1$ points [27, 1.5.3], and as in the previous example, one lets $c$ go to 1. So (PD3) cannot hold anymore. In [26], Tits defines a generalized $n$-gon over $\mathbb{F}_{1}$ to be an ordinary $n$-gon. (The fact that the number of lines incident with a point is also $1+1$, is explained at the end of $\S 1.6$.)

\subsection{Example: projective spaces of higher dimension}

Generalizing the first example to higher dimensions, projective $n$-spaces over $\mathbb{F}_{1}$ should be sets $X$ of cardinality $n+1$ endowed with the geometry of $2^{X}$ : any subset (of cardinality $0 \leq r+1 \leq n+1$ ) is a subspace (of dimension $r$ ). In other words, projective $n$-spaces over $\mathbb{F}_{1}$ are complete graphs on $n+1$ vertices with a natural subspace structure. It is important to note that these spaces still satisfy the Veblen-Young axioms [28], and that they are the only such incidence geometries with thin lines.

In the same vein, combinatorial affine $\mathbb{F}_{1}$-spaces consist of one single point and a number $m$ of one-point-lines through it; $m$ is the dimension of the space. We will come back to this definition in $\S 2$.

In this paper, for any object $X$ in a category $\mathcal{C}, \operatorname{Aut}(X)$ denotes the automorphism group of $X$, and $\mathbf{S}_{m}$ denotes the symmetric group acting on $m$ letters.

In the next proposition, "combinatorial projective space" means "projective $n$ space (over a field $k$ ) seen as a rank $n$ geometry," that is, the geometry of linear subspaces of the vector space $V(n+1, k)$. Its elements set $E$ consists of all the $i$-dimensional vector subspaces, with $i=0,1, \ldots, n$, and the type function

$$
\tau: E \mapsto\{-1,0, \ldots, n-1\}
$$

maps an $i$-dimensional vector subspace, to $i-1$. In the projective context, we call such a space an $(i-1)$-dimensional projective subspace. Incidence is inherited from the vector space. 
Proposition 1.1 (See, e.g., Cohn [4] and Tits [26]). Let $n \in \mathbb{N} \cup\{-1\}$. The combinatorial projective space $\mathbf{P G}\left(n, \mathbb{F}_{1}\right)=\mathbf{P G}(n, 1)$ is the complete graph on $n+1$ vertices endowed with the induced geometry of subsets, and $\operatorname{Aut}\left(\mathbf{P G}\left(n, \mathbb{F}_{1}\right)\right) \cong$ $\mathbf{P G L}_{n+1}\left(\mathbb{F}_{1}\right) \cong \mathbf{S}_{n+1}$.

It is important to note that any $\mathbf{P G}(n, k)$ with $k$ a field contains (many) subgeometries isomorphic to $\mathbf{P G}\left(n, \mathbb{F}_{1}\right)$ as defined above; so the latter object is independent of $k$, and is a common geometric structure of all projective spaces of a fixed given dimension (that is, it is present, as a subgeometry, in each of these spaces). We depict this idea romantically as

$$
\underline{\mathcal{A}}:\{\mathbf{P G}(n, k) \mid k \text { field }\} \longrightarrow\left\{\mathbf{P G}\left(n, \mathbb{F}_{1}\right)\right\} .
$$

More precisely, we imagine $\mathbb{F}_{1}$ to be an initial object in an enlarged category $\mathcal{R}$ of commutative rings, so for each field $k$ we have a diagram with unique morphisms

$$
\mathbb{F}_{1} \longrightarrow \mathbb{Z} \longrightarrow k .
$$

(We suppose the morphism $\mathbb{Z} \longrightarrow k$ still is unique in $\mathcal{R}$.) For each positive integer $n$, consider the category $\mathcal{R}_{n}$ of commutative rings $A\left[X_{0}, \ldots, X_{n}\right]$, with $A$ an object in $\mathcal{R}$, and where the morphisms send, for each $i \in\{0,1, \ldots, n\}$, $X_{i} \mapsto X_{i}$ (and act as ring morphisms on the coefficient rings $A$ ). Applying the Proj-functor on $\mathcal{R}_{n}$ (defined on the enlarged category $\mathcal{R}$ ), we obtain a diagram

$$
\operatorname{Proj}\left(\mathbb{F}_{1}\left[X_{0}, \ldots, X_{n}\right]\right) \longleftarrow \operatorname{Proj}\left(\mathbb{Z}\left[X_{0}, \ldots, X_{n}\right]\right) \longleftarrow \operatorname{Proj}\left(k\left[X_{0}, \ldots, X_{n}\right]\right) .
$$

So we imagine the projective space $\operatorname{Proj}\left(\mathbb{F}_{1}\left[X_{0}, \ldots, X_{n}\right]\right) / \mathbf{P G}\left(n, \mathbb{F}_{1}\right)$ to be a terminal object in the appropriate category.

Further in this paper (in $\S 2$ ), we will formally find the automorphism groups of $\mathbb{F}_{1}$-vector spaces through matrices, and these groups will perfectly agree with Proposition 1.1.

\subsection{Example: buildings}

The examples of the previous subsections can be generalized to all buildings $\mathcal{B}$ : in that case, the $\mathbb{F}_{1}$-copy is an apartment $\mathcal{A}$. In the context of $\mathbb{F}_{1}$-geometry, apartments are often called Weyl geometries. We refer to [26] and [20] for details. One approach in rank $\geq 3$ is to look at a standard BN-pair, and then to consider the geometry induced on the Weyl group of the BN-pair, thus giving an apartment. As we have seen (for projective spaces), one can also start from a point-line characterization of the building (or other geometry), and introduce a 
thin-ness axiom. It is clear that different point-line characterizations of the same class of buildings in principle could lead to essentially different $\mathbb{F}_{1}$-versions. So in this approach, a more general notion of Weyl geometry has to be taken into account. The example below will highlight this idea.

In this section, we will take a deeper look into the polar spaces.

We use the Buekenhout-Shult approach [2] to define polar spaces in a synthetic way. We start from a point-line geometry $\Gamma=(\mathcal{P}, \mathcal{L}, \mathrm{I})$, and we ask for the following axioms:

BS1 $\Gamma$ is a partial linear space.

BS2 ["One-or-all axiom"] If $x$ is a point and $Y$ is a line not incident with $x$, then either $x$ is collinear with precisely one point of $Y$, or with all points of $Y$.

BS3 We have

$$
\operatorname{rad}:=\bigcap_{z \in \mathcal{P}} z^{\perp}=\emptyset ;
$$

here $z^{\perp}$ is the set of points of $\Gamma$ which are incident with some line on $z$.

Suppose that each line is incident with precisely two points (in terms of [3], each line is a short line); this will be our definition of a polar space over $\mathbb{F}_{1}$. We ignore the third degeneracy axiom for now. Then a polar space over $\mathbb{F}_{1}$ is an undirected graph which satisfies (BS2). So, it is a graph with the property that for each set of three distinct vertices there are either 0,2 or 3 edges which contain two vertices of this set. So the complementary graph is a graph with the property that for each set of three distinct vertices there are either 0,1 or 3 edges which contain two vertices of this set. Such a graph is a disjoint union of complete subgraphs; for, if $x$ and $y$ are vertices which lie in a connected component, then by induction, they are adjacent. So the connected components are complete subgraphs ("cliques"). Now by complementing again, we obtain the original graph, so this is a complete multipartite graph: if the connected components in the complement have respectively $n_{1}, \ldots, n_{k}$ vertices, then we obtain a graph isomorphic to $\mathbf{K}_{n_{1}, \ldots, n_{k}}$.

Remark 1.2. This short argument was communicated to me by Andries Brouwer (in April 2017).

The subspace structure of the corresponding polar space is given by considering all the cliques in $\mathbf{K}_{n_{1}, \ldots, n_{k}}$. We keep using the same notation for the corresponding polar space.

The following proposition sums up some of the direct consequences. 
Proposition 1.3. (i) The (possibly degenerate) polar spaces over $\mathbb{F}_{1}$ are precisely the complete multipartite graphs $\mathbf{K}_{n_{1}, \ldots, n_{k}}$, endowed with the natural $\mathbb{F}_{1}$-subspace structure.

(ii) A polar space $\mathbf{K}_{n_{1}, \ldots, n_{k}}$, endowed with the natural $\mathbb{F}_{1}$-subspace structure, is degenerate if and only if some $n_{i}=1$.

(iii) The maximal dimension of a linear subspace of a polar space $\mathbf{K}_{n_{1}, \ldots, n_{k}}$ is $k-1$, and the dimension of the ambient projective space is $\sum_{i=1}^{k} n_{i}-1$.

The automorphism groups of the polar spaces over $\mathbb{F}_{1}$ are easily determined and well known, since they coincide with the automorphism groups of the corresponding complete multipartite graphs. In case $n_{1}=n_{2}=\cdots=n_{k}=2$, we obtain a Coxeter group of type $\mathrm{B}_{k}$; it is isomorphic to the group of signed bijections

$$
\beta:\{ \pm 1, \ldots, \pm k\} \mapsto\{ \pm 1, \ldots, \pm k\} .
$$

The maximal dimension of a linear subspace is then $k-1$.

This particular case is one of the two cases Tits considers in [26]; he also defines another type of polar space over $\mathbb{F}_{1}$, also defined on $\mathbf{K}_{n_{1}=2, n_{2}=2, \ldots, n_{k}=2}$, but so that there are two types of maximal singular subspaces, and the automorphism group is isomorphic to a Coxeter group of type $\mathrm{D}_{k}$. From the viewpoint of above, this consideration seems a bit artificial. (The Buekenhout-Shult approach was published long after Tits's paper [26].)

Usually a polar space of rank $k$ is called thick, if all lines have at least 3 points, and if all $(k-1)$-spaces are on at least 3 maximal singular subspaces; otherwise it is called thin. For the polar spaces $\mathbf{K}_{n_{1}=2, n_{2}=2, \ldots, n_{k}=2}$, besides that every line is short, we also have that every $(k-1)$-space is on precisely 2 maximal singular subspaces. This latter property is of course not true for general $\mathbb{F}_{1}$-polar spaces; however, if at least one $n_{i}=2$, then some $(k-1)$-spaces are in precisely 2 maximal singular subspaces.

It makes the discussion all the more interesting.

Remark 1.4. In [3] polar spaces with short lines are classified. As a polar space of rank 1 is nothing but a set of points, the above examples are direct sums of polar spaces of rank 1 , as in [3, p. 226, (1)].

Remark 1.5. It might be interesting to study the varieties $F\left(\mathbf{K}_{n_{1}, \ldots, n_{k}}\right) \otimes_{\operatorname{Spec}\left(\mathbb{F}_{1}\right)}$ $\operatorname{Spec}(\ell)$, with $\ell$ a field (in the notation of $\S 7$ ). 


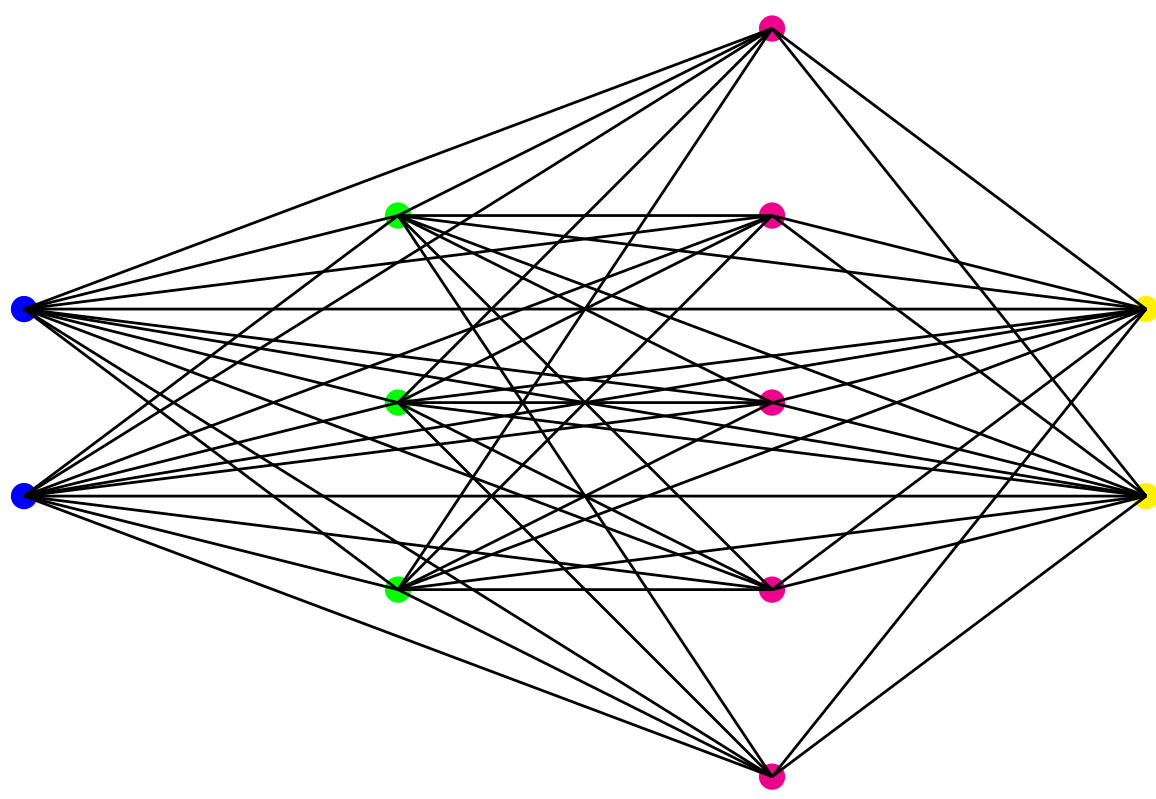

Figure 1: The thin polar space $\mathbf{K}_{2,3,5,2}$.

\subsection{Example: graphs}

Let $\Gamma$ be any graph, and see it as an incidence geometry with the additional property that any line/edge has precisely two distinct points/vertices. (Let us assume for the sake of convenience that it has no loops.) Then over $\mathbb{F}_{1}$, nothing changes, and hence graphs are fixed points of the functor which sends incidence geometries to their $\mathbb{F}_{1}$-models.

\subsection{The functor $\underline{\mathcal{A}}$}

In [20], a functor $\underline{\mathcal{A}}: \mathrm{B} \mapsto \mathrm{A}$ is described which associates to a natural class $\mathrm{B}$ of "combinatorial $\mathbb{F}_{1}$-geometries" its class A of " $\mathbb{F}_{1}$-versions" in much the same way as we have done here for the examples in $\S \S 1.1-1.5$. These $\mathbb{F}_{1}$-versions can be obtained as fixed objects of $\underline{\mathcal{A}}$ (which is called Weyl functor in loc. cit.).

\subsubsection{Intuitive description of $\mathcal{A}$}

The $\mathbb{F}_{1}$-functor $\underline{\mathcal{A}}$ should have several properties (with respect to the images); for the details, we refer to the chapter [20]. Here, we isolate the following fundamental properties which will be useful for the present paper: 
A1- all lines should have at most 2 different points;

A2 - an image should be a "universal object," in the sense that it should be a subgeometry of any thick geometry of the same "type" (defined over any field, if at all defined over one) of at least the same rank;

A3- it should carry the same axiomatic structure (for example: $o \in$ A and elements of $\underline{\mathcal{A}}^{-1}(o)$ carry the same Buekenhout-Tits diagram, cf. $\left.\S 1.6 .2\right)$;

$\mathrm{F}$ - as A will be a subclass of the class of $\mathbb{F}_{1}$-geometries, it should consist precisely of the fixed elements of $\underline{\mathcal{A}}$.

Remark 1.6. We work up to point-line duality: that is why we are allowed to ask, without loss of generality, that lines have at most two points. We do not ask that they have precisely two points, one motivation being e.g. (combinatorial) affine spaces over $\mathbb{F}_{1}$, in which any line has precisely one point.

In some sense, the number of lines through a point of an element $\Gamma$ of A should reflect the rank of the geometries in $\mathcal{A}^{-1}(\Gamma)$. Think for example of the combinatorial affine and projective spaces over $\mathbb{F}_{1}$, and the Weyl geometries of buildings as described by Tits. This principle is a very important feature in the work of Mérida-Angulo and the author described in $§ 7$.

\subsubsection{Some remarks on diagram geometry: Buekenhout-Tits diagrams}

Let $\mathcal{D}$ be a labeled graph on $I$, where for $i, j \in I$ the label $\mathcal{D}_{i j}$ is a class of rank 2 geometries. We say that $\mathcal{D}$ is a Buekenhout-Tits diagram for the geometry $\Gamma=(X, \mathrm{I}, I, t)$ when for every flag $F$ of $\Gamma$ of corank 2, say $t(F)=I \backslash\{i, j\}$, the residue $\Gamma_{F}$ belongs to the class of geometries $\mathcal{D}_{i j}$.

This is a recursive definition for the concept of diagram in terms of what the labeled edges mean for rank 2 geometries.

\subsubsection{Some traditional labels}

We introduce the nomenclature for some frequently used labels.

- $\quad$ Di: Every $i$-object is incident with every $j$-object.

$\longrightarrow \mathrm{A}_{2}$ : The $i$-objects and $j$-objects form the points and lines of an axiomatic projective plane.

$\mathrm{B}_{2}$ : The $i$-objects and $j$-objects form the points and lines of a generalized quadrangle. 
$m \quad \mathrm{I}_{2}(m)(m \in\{6,8\})$ : The $i$-objects and $j$-objects form the points and lines of a generalized hexagon/octagon.

Af Af: The $i$-objects and $j$-objects form the points and lines of an axiomatic affine plane.

C C: The $i$-objects and $j$-objects form the points and edges of a complete graph.

Many other such diagrams are used, but those will be of no concern for our purposes.

\subsubsection{An example}

The geometry of points, lines and planes in a 3-dimensional combinatorial projective space satisfies the axioms given by the diagram

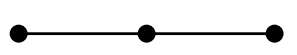

By the result of Veblen-Young [28], combinatorial 3-dimensional projective spaces necessarily come from (left or right) vector spaces over a skew field. It is an easy exercise to prove the Veblen-Young axiom from the $\mathrm{A}_{n}$-diagram, so that the following holds.

Theorem 1.7. A (thick) Buekenhout-Tits geometry satisfying the $\mathrm{A}_{n}$-diagram axioms is a projective space.

So the axioms which are imposed on the Buekenhout-Tits geometry by the $\mathrm{A}_{n}$-diagram suffice to fully determine the incidence geometry.

\subsubsection{Low rank}

Our natural starting point in [25] was the category of Buekenhout-Tits geometries; all buildings are members. We only consider connected geometries - the general theory can be reduced to the connected theory as usual. We call this assumption "C."

The first step is to classify the elements of A. We take A1-A2-A3-A4-C to be the main axioms. After having determined $\mathrm{A}$, one defines the functor $\mathcal{A}$, and the inverse image $\mathcal{A}^{-1}(\mathrm{~A})$ in $\mathrm{BT}$, the category of Buekenhout-Tits geometries with obvious morphisms. This inverse image is denoted by $\mathrm{BT}_{\mid 1}$.

We include some detailed comments about the rank 1 and 2 cases. 


\section{Rank 1 - description of $\mathrm{A}$}

- $\quad$ - $\quad \mathrm{Di}_{1}$ : Every $i$-object is incident with every $j$-object. Over $\mathbb{F}_{1}$, this example has one line and one point, and they are incident.

$\longrightarrow \quad \mathrm{A}_{1}$ : The $i$-objects and $j$-objects form the points and lines of a combinatorial projective line over $\mathbb{F}_{1}$ : two distinct points incident with a line.

Af $\longrightarrow$ Af: The $i$-objects and $j$-objects form the points and lines of a combinatorial affine line over $\mathbb{F}_{1}$ : one point incident with one line (the "absolute flag").

\section{Rank 2 - description of A}

The rank 2 examples of Buekenhout-Tits geometries are the most important ones, since all other examples (ignoring the rank 0 and 1 cases) are constructed from them through axioms governed by the diagrams. By A4, any point is incident with at most two lines. Taking this property into account, the reader easily sees that the geometries must be of one of the following types (where at the end, we introduce a new type).

- $\quad-\quad \mathrm{Di}_{2}$ : Every $i$-object is incident with every $j$-object. Over $\mathbb{F}_{1}$, this example has two lines and two points, and any point is incident with any line.

$\longrightarrow \quad \mathrm{A}_{2}$ : The $i$-objects and $j$-objects form the points and lines of a combinatorial projective plane over $\mathbb{F}_{1}$ : an ordinary triangle.

$\longrightarrow \quad \mathrm{B}_{2}$ : The $i$-objects and $j$-objects form the points and lines of a generalized quadrangle over $\mathbb{F}_{1}$, which is an ordinary 4-gon (the polar space $\mathbf{K}_{2,2}$ ).

• $\quad \mathrm{I}_{2}(m)(m \in \mathbb{N} \cup\{\infty\}, m \geq 5)$ : The $i$-objects and $j$-objects form the points and lines of an ordinary $m$-gon.

- Af Af: The $i$-objects and $j$-objects form the points and lines of a combinatorial affine plane over $\mathbb{F}_{1}$ : one point incident with two lines which are incident with only that point.

There is also an odd-one-out class of examples which enters the picture.

$\mathrm{U}$

$\mathrm{U}$ : The $i$-objects and $j$-objects form the points and lines of a connected loose tree of valency $\leq 2$, with at least one end point or end edge. (Lines with one point are allowed, so at the ends, one can have end points or end lines; we call trees with this relaxation "loose trees" - see §7.) 
The unique examples of $\mathrm{Di}_{2}, \mathrm{~A}_{2}, \mathrm{~B}_{2}$ and $\mathrm{I}_{2}(m)$ are self-dual. The class described by $U$ is also self-dual, while the point-line dual of the Af-type geometry is one of the rank 1 examples. The example in Af is contained in $U$ but plays a different role in this discussion. Also note that $\mathrm{I}_{2}(\infty) \cup \mathrm{U}$ precisely is the class of loose trees with valency $\leq 2$.

\section{Rank 2 - description of BT}

Pulling back these examples to BT (by $\underline{\mathcal{A}}^{-1}$ ), it is clear — taking A3 into account - that

$\star \mathcal{A}^{-1}(\cdot)$, with values taken in $\mathrm{Di}_{2} \cup \mathrm{A}_{2} \cup \mathrm{B}_{2} \cup \mathrm{I}_{2}(m) \cup \mathrm{Af}$, respectively consists of the generalized digons, projective planes, generalized quadrangles, generalized $m$-gons, and affine planes. We remark that in all of these cases, the corresponding elements in A are also assumed to be in the fibers (thin examples are allowed in $\mathcal{A}^{-1}(\cdot)$; cf. property F)! In the case $m=\infty$, $\underline{\mathcal{A}}^{-1}\left(\mathrm{I}_{2}(\infty)\right)$ is the class of trees without end points (where more than two points per line are allowed).

$\star \mathcal{A}^{-1}(\mathrm{U})$ is the set of loose trees with end points or end edges (where more than two points per line are allowed).

In the case of generalized polygons $\Gamma$, that is, elements of

$$
\underline{\mathcal{A}}^{-1}\left(\mathrm{Di}_{2} \cup \mathrm{A}_{2} \cup \mathrm{B}_{2} \cup \mathrm{I}_{2}(m)\right),
$$

any pair of elements in $\mathcal{P} \cup \mathcal{B}$ is contained in a geometry isomorphic to $\mathcal{A}(\Gamma)$, making them in a sense the most natural geometries defined over $\mathbb{F}_{1}$. It might be desirable to impose similar conditions on elements of $\underline{\mathcal{A}}^{-1}(\mathrm{U})$.

We denote the class of $\mathbb{F}_{1}$-Buekenhout-Tits geometries defined in this section by $\mathrm{BT}_{\mid 1}^{(2)}$, and its image in $\mathrm{A}$ by $\mathrm{A}^{(2)}$.

Remark 1.8. Note that all polar spaces $\mathbf{K}_{n_{1}, n_{2}}$ with $n_{1}, n_{2} \leq 2$ are elements of $\mathrm{A}$.

\section{Combinatorial theory}

Certain invariants attached to a finite field $k$ happen to be expressible as a value $p(|k|)$ for some polynomial $p \in \mathbb{Z}[X]$. So it makes sense to replace the argument with 1 and ask for the meaning of the resulting value $p(1)$. 
It is then easy to see the symmetric group also directly as a "limit" with $|k| \longrightarrow 1$ of linear groups $\mathbf{P G}(n, k)$ (with the dimension fixed). The number of elements in $\mathbf{P G}(n, k)$ (where $k=\mathbb{F}_{q}$ is assumed to be finite and $q$ is a prime power) is

$$
\frac{\left(q^{n+1}-1\right)\left(q^{n+1}-q\right) \cdots\left(q^{n+1}-q^{n}\right)}{(q-1)}=(q-1)^{n} N(q)
$$

for some polynomial $N(X) \in \mathbb{Z}[X]$, and we have

$$
N(1)=(n+1) !=\left|\mathbf{S}_{n+1}\right| .
$$

Now let $n, q \in \mathbb{N}$, and define $[n]_{q}=1+q+\cdots+q^{n-1}$. (For $q$ a prime power, $[n]_{q}=|\mathbf{P G}(n, q)|$.) Put $[0]_{q} !=1$, and define

$$
[n]_{q} !:=[1]_{q}[2]_{q} \ldots[n]_{q}
$$

and

$$
\left[\begin{array}{l}
n \\
k
\end{array}\right]_{q}=\frac{[n]_{q} !}{[k]_{q} ![n-k]_{q} !} .
$$

If $q$ is a prime power, this is the number of $(k-1)$-dimensional subspaces of $\mathbf{P G}(n-1, q)\left(=\left|\operatorname{Grass}(k, n)\left(\mathbb{F}_{q}\right)\right|\right)$. The next proposition again gives sense to the limit situation of $q$ replaced by 1 .

Proposition 2.1 (See e.g. Cohn [4]). The number of $k$-dimensional subspaces of $\mathbf{P G}\left(n, \mathbb{F}_{1}\right)$, with $k \leq n \in \mathbb{N}$, equals

$$
\left[\begin{array}{l}
n+1 \\
k+1
\end{array}\right]_{1}=\frac{(n+1) !}{(n-k) !(k+1) !}=\left(\begin{array}{l}
n+1 \\
k+1
\end{array}\right) .
$$

Many other enumerative formulas in Linear Algebra, Projective Geometry, etc. over finite fields $\mathbb{F}_{q}$ seem to keep meaningful interpretations if $q$ is replaced by 1 , and this phenomenon (the various interpretations) suggests a deeper theory in characteristic one.

Right now, we will have a look at some Linear Algebra features in characteristic 1. Many of them are taken from Kapranov and Smirnov's [12].

\subsection{A definition for $\mathbb{F}_{1}$}

We define $\mathbb{F}_{1}$ as the monoid $\{0,1\}$ for which we only have the following operations:

$$
0 \cdot 1=0=0 \cdot 0 \text { and } 1 \cdot 1=1 .
$$


This setting makes $\mathbb{F}_{1}$ sit in between the group $(\{\mathbf{1}\}, \cdot)$ and $\mathbb{F}_{2}$. So in absolute Linear Algebra we are not allowed to have addition of vectors and we have to define everything in terms of scalar multiplication.

The reason why this approach is natural, will become clear when we consider, e.g., linear automorphisms later in this section.

\subsection{Field extensions of $\mathbb{F}_{1}$}

For each $m \in \mathbb{N}^{\times}$we define the field extension $\mathbb{F}_{1^{m}}$ of $\mathbb{F}_{1}$ of degree $m$ as the monoid $\{0\} \cup \mu_{m}$, where $\mu_{m}$ is the (multiplicatively written) cyclic group of order $m$, and 0 is an absorbing element for the extended multiplication to $\{0\} \cup$ $\mu_{m}$.

\subsection{Vector spaces over $\mathbb{F}_{1(n)}$}

At the level of $\mathbb{F}_{1}$ we cannot make a distinction between affine spaces and vector spaces - as a torsor, nothing happens. This motivates the following definition: a vector/affine space over $\mathbb{F}_{1^{n}}, n \in \mathbb{N}^{\times}$, is a triple $V=\left(\mathbf{0}, X, \mu_{n}\right)$, where $\mathbf{0}$ is a point which is sometimes called "the distinguished point" and $X$ a set, and where $\mu_{n}$ acts freely on $X$. Each $\mu_{n}$-orbit corresponds to a direction. If $n=1$, we get the notion considered in $\S 1.3$. If the dimension is countably infinite, $\mu_{n}$ may be replaced by $\mathbb{Z},+$ (the infinite cyclic group). Another definition is needed when the dimension is larger.

\subsection{Basis}

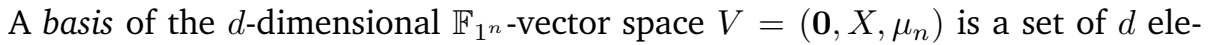
ments in $X$ that is a set of representatives of the $\mu_{n}$-action; here, formally, $X$ consists of $d n$ elements, and $\mu_{n}$ is the cyclic group with $n$ elements. (If $d$ is not finite one selects exactly one element in each $\mu_{n}$-orbit.) If $n=1$, we only have $d$ elements in $X$ (which expresses the fact that the $\mathbb{F}_{1}$-linear group indeed is the symmetric group).

Once a choice of a basis $\left\{b_{i} \mid i \in I\right\}$ has been made, any element $v$ of $V$ can be uniquely written as $b_{j}^{\alpha^{u}}$, for unique $j \in I$ and $\alpha^{u} \in \mu_{n}=\langle\alpha\rangle$. So we can also represent $v$ in coordinates by a $d$-tuple with exactly one nonzero entry, namely $b_{j}^{\alpha^{u}}$ (in the $j$-th column). 


\subsection{Dimension}

We define the dimension of $V$ to be given by $\operatorname{card}(V) / n=d$ (the number of $\mu_{n}$-orbits).

\subsection{Field extension}

Let $V=\left(\mathbf{0}, X, \mu_{n}\right)$ be a (not necessarily finite dimensional) vector space of dimension $d$ over $\mathbb{F}_{1^{n}}, n$ finite, so that $\left|X=X_{V}\right|=d n$. For any positive divisor $m$ of $n$, with $n=m r, V$ can also be seen as a $d r$-space over $\mathbb{F}_{1^{m}}$. Note that there is a unique cyclic subgroup $\mu_{m}$ of $\mu_{n}$ of size $m$, so there is only one way to see $V$ naturally as a $d r$-space in this context (since we have to preserve the structure of $V$ in the process).

\subsection{Projective completion}

By definition, the projective completion of an affine space $\mathbf{A G}(n, k), n \in \mathbb{N}$ and $k$ a field, is the projective space $\mathbf{P G}(n, k)$ of the same dimension and defined over the same field, which one obtains by adding a hyperplane at infinity.

We have seen how to perform projective completion over $\mathbb{F}_{1}$ through the following formula:

$$
\mathbf{P G}\left(n, \mathbb{F}_{1}\right)=\mathbf{A G}\left(n, \mathbb{F}_{1}\right)+\mathbf{P G}\left(n-1, \mathbb{F}_{1}\right)
$$

Here we use typical motivic notation (e.g., it is one of the standard expressions in the Grothendieck ring of varieties if one replaces $\mathbb{F}_{1}$ by a field $k$ ) to express affine space as a piece of projective space (of the same dimension).

If one replaces $\mathbb{F}_{1}$ by an extension $\mathbb{F}_{1^{m}}$, the story is more complicated - see e.g. $[20,24]$.

\subsection{Linear automorphisms}

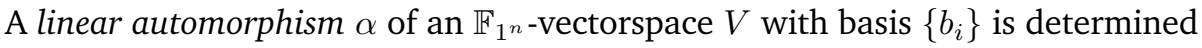
by the base images

$$
\alpha\left(b_{i}\right)=b_{\sigma(i)}^{\beta_{i}}
$$

for some power $\beta_{i}$ of the primitive $n$-th root of unity $\alpha$, and some permutation $\sigma \in \mathbf{S}_{d}$. Then we have that

$$
\mathbf{G L}_{d}\left(\mathbb{F}_{1^{n}}\right) \cong\left(\mu_{n}\right)^{d} \rtimes \mathbf{S}_{d} .
$$


Elements of $\mathbf{G L}_{d}\left(\mathbb{F}_{1^{n}}\right)$ can be written as $(d \times d)$-matrices with precisely one element of $\mu_{n}$ in each row or column (and conversely, any such element determines an element of $\left.\mathbf{G L}_{d}\left(\mathbb{F}_{1^{n}}\right)\right)$. In this setting, $\mathbf{S}_{d}$ is represented by $(d \times d)$-matrices with in each row and column exactly one 1 - permutation matrices.

Remark 2.2. Note that the underlying reason that rows and columns have only one nonzero element is that we do not have addition in our vector space.

\section{Deninger-Manin theory}

In a number of works $[8,9,10]$ on motives and regularized determinants, Deninger played with the possibility of translating Weil's proof of the Riemann Hypothesis for function fields of projective curves over finite fields $\mathbb{F}_{q}$ to the hypothetical curve $\overline{\operatorname{Spec}(\mathbb{Z})}$. This idea also occurred, for instance, in Haran [11], and circulated in work of Smirnov [17] — see [22]. In [9], Deninger gave a description of conditions on a certain category M of motives which might allow such a translation.

Let $\mathcal{C}$ be a nonsingular absolutely irreducible projective algebraic curve over the finite field $\mathbb{F}_{q}$. Fix an algebraic closure $\overline{\mathbb{F}_{q}}$ of $\mathbb{F}_{q}$ and let $m \neq 0$ be a positive integer; we have the following Lefschetz formula for the number $\left|\mathcal{C}\left(\mathbb{F}_{q^{m}}\right)\right|$ of rational points over $\mathbb{F}_{q^{m}}$ :

$$
\left|\mathfrak{C}\left(\mathbb{F}_{q^{m}}\right)\right|=\sum_{\omega=0}^{2}(-1)^{\omega} \operatorname{Tr}\left(\operatorname{Fr}^{m} \mid H^{\omega}(\mathcal{C})\right)=1-\sum_{j=0}^{2 g} \lambda_{j}^{m}+q^{f},
$$

where Fr is the Frobenius endomorphism acting on the étale $\ell$-adic cohomology of $\mathcal{C}$, the $\lambda_{j}$ s are the eigenvalues of this action, and $g$ is the genus of the curve. We then have a motivic weight decomposition

$$
\begin{aligned}
\zeta_{\mathfrak{C}}(s) & =\prod_{\omega=0}^{2} \zeta_{h^{\omega}(\mathcal{C})}(s)^{(-1)^{\omega-1}}=\frac{\prod_{j=1}^{2 g}\left(1-\lambda_{j} q^{-s}\right)}{\left(1-q^{-s}\right)\left(1-q^{1-s}\right)} \\
& =\frac{\operatorname{DET}\left(\left(s \cdot \mathbf{1}-q^{-s} \cdot \operatorname{Fr}\right) \mid H^{1}(\mathcal{C})\right)}{\operatorname{DET}\left(\left(s \cdot \mathbf{1}-q^{-s} \cdot \operatorname{Fr}\right) \mid H^{0}(\mathcal{C})\right) \operatorname{DET}\left(\left(s \cdot \mathbf{1}-q^{-s} \cdot \operatorname{Fr}\right) \mid H^{2}(\mathcal{C})\right)} .
\end{aligned}
$$

(Here the $\omega$-weight component is the zeta function of the pure weight $\omega$ motive $h^{\omega}(\mathcal{C})$ of $\left.\mathcal{C}.\right)$

The following analogous formula would hold in $\mathrm{M}$, where $\mathcal{C}$ is replaced by the 
"curve" $\overline{\operatorname{Spec}(\mathbb{Z})}$ :

$$
\begin{aligned}
& \zeta_{\overline{\operatorname{Spec}(\mathbb{Z})}}(s)=2^{-1 / 2} \pi^{-s / 2} \Gamma\left(\frac{s}{2}\right) \zeta(s)=\frac{\prod_{\rho} \frac{s-\rho}{2 \pi}}{\frac{s}{2 \pi} \frac{s-1}{2 \pi}} \stackrel{?}{=} \\
& \frac{\operatorname{DET}\left(\frac{1}{2 \pi}(s \cdot \mathbf{1}-\varrho) \mid H^{1}\left(\overline{\operatorname{Spec}(\mathbb{Z})}, *_{\text {abs }}\right)\right)}{\operatorname{DET}\left(\frac{1}{2 \pi}(s \cdot \mathbf{1}-\varrho) \mid H^{0}\left(\overline{\operatorname{Spec}(\mathbb{Z})}, *_{\text {abs }}\right)\right) \operatorname{DET}\left(\frac{1}{2 \pi}(s \cdot \mathbf{1}-\varrho) \mid H^{2}\left(\overline{\operatorname{Spec}(\mathbb{Z})}, *_{\text {abs }}\right)\right)} .
\end{aligned}
$$

(The notation used in (20) is as follows:

$* \square$ is the infinite regularized product;

* DET denotes the regularized determinant;

* $\varrho$ is an "absolute" Frobenius endomorphism;

* the $H^{i}(\overline{\operatorname{Spec}(\mathbb{Z})}, *$ abs $)$ are certain proposed cohomology groups, and

* the $\rho$ s run through the set of critical zeroes of the classical Riemann zeta.)

Note that in the left-hand side of (20), we consider $\overline{\operatorname{Spec}(\mathbb{Z})}$ instead of $\operatorname{Spec}(\mathbb{Z})$, because we want to have a projective curve as in the expression for the motivic weight decomposition of $\mathcal{C}$. This is why the factor

$$
2^{-1 / 2} \pi^{-s / 2} \Gamma\left(\frac{s}{2}\right)
$$

occurs - it is the zeta-factor at infinity.

Conjecturally, in M there are motives $h^{0}$ ("the absolute point"), $h^{1}$ and $h^{2}$ ("the absolute Lefschetz motive") with zeta functions

$$
\zeta_{h^{w}}(s)=\operatorname{DET}\left(\frac{1}{2 \pi}(s \cdot \mathbf{1}-\varrho) \mid H^{w}\left(\overline{\operatorname{Spec}(\mathbb{Z})} *_{\text {abs }}\right)\right)
$$

for $w=0,1,2$. Deninger computed that $\zeta_{h^{0}}(s)=s / 2 \pi$ and $\zeta_{h^{2}}(s)=(s-1) / 2 \pi$. Manin proposed to interpret $h^{0}$ as $\operatorname{Spec}\left(\mathbb{F}_{1}\right)$ and $h^{2}$ as the affine line over $\mathbb{F}_{1}$, in [15].

In [15], Manin then suggested to develop Algebraic Geometry over the field with one element, already in this specific context. So what is a scheme over $\mathbb{F}_{1}$ ? 


\section{Deitmar schemes}

One of the first papers which systematically studied a scheme theory over $\mathbb{F}_{1}$ was Deitmar's [5], published in 2005. The study in [5] is related to Kato's paper [13]; see $\S 5$ and $\S 9$ of that paper. By that time, Soulé had already published his fundamental $\mathbb{F}_{1}$-approach to varieties [18].

In $\mathbb{Z}$-scheme theory, a scheme $X$ is a locally ringed topological space which is locally isomorphic to affine schemes. That is to say, $X$ is covered by opens $\left\{U_{i} \mid i \in I\right\}$ such that the restriction of the structure sheaf $\mathcal{O}_{X}$ to each $U_{j}$ is itself a locally ringed space which is isomorphic to the spectrum of a commutative ring. When aiming at an Algebraic Geometry over $\mathbb{F}_{1}$, one wants to have similar definitions at hand, but the commutative rings have to be replaced by appropriate algebraic structures which reflect the $\mathbb{F}_{1}$-nature.

Several attempts have been made to define schemes "defined over $\mathbb{F}_{1}$," and often the approaches only differ in subtle variations. We only need the most basic one, which is the "monoidal scheme theory" of Anton Deitmar [6]. In this theory, the role of commutative rings over $\mathbb{F}_{1}$ is played by commutative monoids (with a zero).

\subsection{Rings over $\mathbb{F}_{1}$}

A monoid is a set $A$ with a binary operation $\cdot: A \times A \longrightarrow A$ which is associative, and has an identity element (denoted 1 ). Homomorphisms of monoids preserve units, and for a monoid $A, A^{\times}$will denote the group of invertible elements (so that if $A$ is a group, $A^{\times}=A$ ).

In [5], Deitmar defines the category of rings over $\mathbb{F}_{1}$ to be the category of monoids (as thus ignoring additive structure), and the category of commutative $\mathbb{F}_{1}$-rings to be the category of commutative monoids. Usually, we will assume without further notice that an $\mathbb{F}_{1}$-ring $A$ also has a zero-element 0 such that $0 \cdot a=0=a \cdot 0, \forall a \in A$.

Below, all monoids will assumed to be abelian.

\subsection{Algebraic closure}

A monoid $A$ is algebraically closed if every equation of the form $x^{n}=a$ with $a \in A$ and $n \in \mathbb{N} \backslash\{0\}$ has $n$ solutions in $A$. Every monoid can be embedded into an algebraically closed monoid, and if $A$ is a group, then there exists a "smallest" such embedding which is called the algebraic closure of $A$. 
The algebraic closure $\overline{\mathbb{F}_{1}}$ of $\mathbb{F}_{1}$ is the group $\mu_{\infty}$ of all complex roots of unity; it is isomorphic to $\mathbb{Q} / \mathbb{Z}$. Note that the multiplicative group $\overline{\mathbb{F}}_{p} \times$ of the algebraic closure $\overline{\mathbb{F}_{p}}$ of the prime field $\mathbb{F}_{p}$ is isomorphic to the group of all complex roots of unity of order prime to $p$, so that the definition of $\overline{\mathbb{F}_{1}}$ might seem strange if compared with the finite field case. One can easily find "meta-arithmetic" arguments to deal with this matter - see [21].

\subsection{Localization}

Let $S$ be a submonoid of the monoid $A$. We define the monoid $S^{-1} A$, the localization of $A$ by $S$, to be

$$
A \times S / \sim
$$

where the equivalence relation " $\sim$ " is given by

$$
(a, s) \sim\left(a^{\prime}, s^{\prime}\right) \text { if and only if } s^{\prime \prime} s^{\prime} a=s^{\prime \prime} s a^{\prime} \text { for some } s^{\prime \prime} \in S .
$$

Multiplication in $S^{-1} A$ is componentwise, and one suggestively writes $\frac{a}{s}$ for the element in $S^{-1} A$ corresponding to $(a, s)$ (so $\frac{a}{s} \cdot \frac{a^{\prime}}{s^{\prime}}=\frac{a a^{\prime}}{s s^{\prime}}$ ).

\subsection{Ideal and spectrum}

If $C$ and $D$ are subsets of the monoid $A, C D$ denotes the set of products $c d$, with $c \in C$ and $d \in D$.

Recall that a monoid is supposed to be abelian. If $C$ is a monoid, $\mathbb{Z}[C]$ denotes the corresponding "monoidal ring" - it is naturally defined similarly to a group ring.

An ideal $\mathfrak{a}$ of a monoid $M$ is a subset such that $M \mathfrak{a} \subseteq \mathfrak{a}$. For any ideal $\mathfrak{a}$ in $M$, $\mathbb{Z}[\mathfrak{a}]$ is an ideal in $\mathbb{Z}[M]$. Note that if $A$ and $B$ are monoids and $\alpha: A \longrightarrow B$ is a morphism, then $\alpha^{-1}(\mathfrak{a})$ is an ideal in $A$ if $\mathfrak{a}$ is an ideal in $B$.

An ideal $\mathfrak{p}$ is called a prime ideal if $S_{\mathfrak{p}}:=M \backslash \mathfrak{p}$ is a monoid (that is, if $u v \in \mathfrak{p}$, then $u \in \mathfrak{p}$ or $v \in \mathfrak{p}$ ). For any prime ideal $\mathfrak{p}$ in $M$, denote by $M_{\mathfrak{p}}=S_{\mathfrak{p}}^{-1} M$ the localization of $M$ at $\mathfrak{p}$. It is easy to prove that the natural map

$$
M \longrightarrow M_{\mathfrak{p}}, m \longrightarrow \frac{m}{1}
$$

with $\mathfrak{p}=M \backslash M^{\times}$is an isomorphism.

Let $M$ be a monoid. The spectrum $\operatorname{Spec}(M)$ of $M$ is the set of prime ideals endowed with the obvious Zariski topology. Note that the spectrum cannot be 
empty since $M \backslash M^{\times}$is a prime ideal. The closed subsets are the empty set and all sets of the form

$$
V(\mathfrak{a}):=\{\mathfrak{p} \in \operatorname{Spec}(M) \mid \mathfrak{a} \subseteq \mathfrak{p}\},
$$

where $\mathfrak{a}$ is any ideal. The point $\eta=\emptyset$ is contained in every nonempty open set and the point $M \backslash M^{\times}$is closed and contained in every nonempty closed set. Note also that for every $m \in M$ the set $V(m):=\{\mathfrak{p} \in \operatorname{Spec}(M) \mid m \in \mathfrak{p}\}$ is closed (as $V(m)=V(M m)$ ).

Proposition 4.1. $M \backslash M^{\times}$is the unique maximal ideal for any monoid $M$, so any such $M$ is a local $\mathbb{F}_{1}$-ring.

\subsection{Structure sheaf}

Let $A$ be a ring over $\mathbb{F}_{1}$. For any open set $U \subseteq \operatorname{Spec}(A)$, one defines $\mathcal{O}_{\operatorname{Spec}(A)}(U)=$ $\mathcal{O}(U)$ to be the set of functions (called sections)

$$
s: U \longrightarrow \coprod_{\mathfrak{p} \in U} A_{\mathfrak{p}}
$$

for which $s(\mathfrak{p}) \in A_{\mathfrak{p}}$ for each $\mathfrak{p} \in U$, and such that there exists a neighborhood $V$ of $\mathfrak{p}$ in $U$, and elements $a, b \in A$, for which $b \notin \mathfrak{q}$ for every $\mathfrak{q} \in V$, and $s(\mathfrak{q})=\frac{a}{b}$ in $A_{\mathfrak{q}}$. The map

$$
\mathcal{O}_{\operatorname{Spec}(A)}: \operatorname{Spec}(A) \longrightarrow \text { monoids }: U \longrightarrow \mathcal{O}(U)
$$

is the structure sheaf of $\operatorname{Spec}(A)$.

Proposition 4.2 (Deitmar [6]). (i) For each $\mathfrak{p} \in \operatorname{Spec}(A)$, the stalk $\mathcal{O}_{\mathfrak{p}}$ of the structure sheaf is isomorphic to the localization of $A$ at $\mathfrak{p}$.

(ii) For global sections, we have $\Gamma(\operatorname{Spec}(A), \mathcal{O}):=\mathcal{O}(\operatorname{Spec}(A)) \cong A$.

\subsection{Monoidal spaces}

A monoidal space is a topological space $X$ together with a sheaf of monoids $\mathcal{O}_{X}$. Call a morphism of monoids $\beta: A \longrightarrow B$ local if $\beta^{-1}\left(B^{\times}\right)=A^{\times}$. A morphism between monoidal spaces $\left(X, \mathcal{O}_{X}\right)$ and $\left(Y, \mathcal{O}_{Y}\right)$ is defined naturally: it is a pair $\left(f, f^{\#}\right)$ with $f: X \longrightarrow Y$ a continuous function, and

$$
f^{\#}: \mathcal{O}_{Y} \longrightarrow f_{*} \mathcal{O}_{X}
$$

a morphism between sheaves of monoids on $Y$. (Here, $f_{*} \mathcal{O}_{X}$, the direct image sheaf on $Y$ induced by $f$, is defined by $f_{*} \mathcal{O}_{X}(U):=\mathcal{O}_{X}\left(f^{-1}(U)\right)$ for all open $U \subseteq Y$.) The morphism is local if each of the induced morphisms $f_{x}^{\#}$ : $\mathcal{O}_{Y, f(x)} \longrightarrow \mathcal{O}_{X, x}$ is local. 
Proposition 4.3 (Deitmar [6]). (i) If $A$ is any $\mathbb{F}_{1}$-ring, we have that the pair $\left(\operatorname{Spec}(A), \mathcal{O}_{A}\right)$ defines a monoidal space.

(ii) If $\alpha: A \longrightarrow B$ is a morphism of monoids, then $\alpha$ induces a morphism of monoidal spaces

$$
\left(f, f^{\#}\right): \operatorname{Spec}(B) \longrightarrow \operatorname{Spec}(A),
$$

yielding a functorial bijection

$$
\operatorname{Hom}(A, B) \cong \operatorname{Hom}_{\text {loc }}(\operatorname{Spec}(B), \operatorname{Spec}(A)),
$$

where on the right-hand side we only consider local morphisms (hence the notation).

\subsection{Deitmar's $\mathbb{F}_{1}$-schemes}

As in the theory of rings, we have defined a structure sheaf $\mathcal{O}_{X}$ on the topological space $X=\operatorname{Spec}(M)$, with $M$ a commutative monoid (with a zero). We define a scheme over $\mathbb{F}_{1}$ to be a topological space together with a sheaf of monoids, locally isomorphic to spectra of monoids in the above sense. The details are below.

Affine schemes. An affine scheme over $\mathbb{F}_{1}$ is a monoidal space which is isomorphic to $\operatorname{Spec}(A)$ for some monoid $A$. Such schemes are coined with the term affine Deitmar schemes or also $\mathcal{D}$-schemes or $\mathcal{D}_{0}$-schemes. (The "DD" stands for "Deitmar"; sometimes the sub-index 0 is added to stress that monoids have a zero in this context.)

General schemes. A monoidal space $X$ is a scheme over $\mathbb{F}_{1}$ if for every point $x \in X$ there is an open neighborhood $U \subseteq X$ such that $\left(U, \mathcal{O}_{X \mid U}\right)$ is an affine scheme over $\mathbb{F}_{1}$. As in the affine case, we also speak of $\mathcal{D}$-schemes and $\mathcal{D}_{0}$-schemes.

A morphism of $\mathcal{D}_{(0)}$-schemes is a local morphism of monoidal spaces. A point $\eta$ of a topological space is a generic point if it is contained in every nonempty open set.

Proposition 4.4 (Deitmar [6]). (i) Any connected $\mathcal{D}_{0}$-scheme has a unique generic point $\emptyset$, and morphisms between connected schemes map generic points to generic points.

(ii) For an arbitrary $\mathcal{D}_{0}$-scheme $X, \operatorname{Hom}\left(\operatorname{Spec}\left(\mathbb{F}_{1}\right), X\right)$ can be identified with the set of connected components of $X$. 


\section{Acquiring flesh (1)}

Given an $\mathbb{F}_{1}$-ring $A$, Deitmar base extension to $\mathbb{Z}$ is defined by

$$
A \otimes \mathbb{Z}=A \otimes_{\mathbb{F}_{1}} \mathbb{Z}=\mathbb{Z}[A] .
$$

Denote the functor of base extension by $\mathcal{F}\left(\cdot, \otimes_{\mathbb{F}_{1}} \mathbb{Z}\right)$.

Conversely, we have a forgetful functor $\mathcal{F}$ which maps any (commutative) ring (with unit) to its (commutative) multiplicative monoid.

Theorem 5.1 (Deitmar [5]). The functor $\mathcal{F}\left(\cdot, \otimes_{\mathbb{F}_{1}} \mathbb{Z}\right)$ is left adjoint to $\mathcal{F}$, that is, for every ring $R$ and every $\mathbb{F}_{1}$-ring $A$ we have that

$$
\operatorname{Hom}_{\text {Rings }}\left(A \otimes_{\mathbb{F}_{1}} \mathbb{Z}, R\right) \cong \operatorname{Hom}_{\mathbb{F}_{1}}(A, \mathcal{F}(R)) .
$$

One obtains a functor

$$
X \longrightarrow X_{\mathbb{Z}}
$$

from $\mathcal{D}_{0}$-schemes to $\mathbb{Z}$-schemes, thus extending the base change functor in the following way: (a) write a scheme $X$ over $\mathbb{F}_{1}$ as a union of affine $\mathcal{D}_{0}$-schemes, $X=\cup_{i} \operatorname{Spec}\left(A_{i}\right)$; (b) then map it to $\cup_{i} \operatorname{Spec}\left(A_{i} \otimes_{\mathbb{F}_{1}} \mathbb{Z}\right)$ (glued via the gluing maps from $X$ ).

Similarly to the general case, we say that the $\mathcal{D}_{0}$-scheme $X$ is of finite type if it has a finite covering by affine schemes $U_{i}=\operatorname{Spec}\left(A_{i}\right)$ such that the $A_{i}$ are finitely generated.

Proposition 5.2 (Deitmar [6]). $X$ is of finite type over $\mathbb{F}_{1}$ if and only if $X_{\mathbb{Z}}$ is a $\mathbb{Z}$-scheme of finite type.

Conversely, one has a functor from monoids to rings, and it is left adjoint to the forgetful functor that sends a ring $(R,+, \times)$ to the multiplicative monoid $(R, \times)$. A scheme $X$ over $\mathbb{Z}$ can be written as a union of affine schemes

$$
X=\cup_{i} \operatorname{Spec}\left(A_{i}\right)
$$

for some set of rings $\left\{A_{i}\right\}$. Then map $X$ to $\cup_{i} \operatorname{Spec}\left(A_{i}, \times\right)$ (using the gluing maps from $X$ ) to obtain a functor from schemes over $\mathbb{Z}$ to schemes over $\mathbb{F}_{1}$ which extends the aforementioned forgetful functor.

The next theorem, which is due to Deitmar, shows that integral $\mathcal{D}_{0}$-schemes of finite type become toric varieties, once pulled to $\mathbb{C}$. (We won't define toric varieties here; we refer the reader to any standard text on these structures. Details can also be found in [21].) 
Theorem 5.3 (Deitmar [7]). Let $X$ be a connected integral $\mathcal{D}_{0}$-scheme of finite type. Then every irreducible component of $X_{\mathbb{C}}$ is a toric variety. The components of $X_{\mathbb{C}}$ are mutually isomorphic as toric varieties.

Other scheme theories over $\mathbb{F}_{1}$ are known for which the base change functor to $\mathbb{Z}$ is "more general." We refer to the monograph [23], and the chapters therein, for a garden of such scheme theories.

\section{Kurokawa theory}

One of the main tools to understand $\mathbb{F}_{1}$-schemes are their zeta functions. In this section, we define the Kurokawa zeta function, and we mention some interesting results taken from [14]. We first start with collecting some basic notions on arithmetic zeta functions.

\subsection{Arithmetic zeta functions}

A $\mathbb{Z}$-variety $X$ is by definition a scheme of finite type over $\mathbb{Z}$. This means that $X$ has a finite covering of affine $\mathbb{Z}$-schemes $\operatorname{Spec}\left(A_{i}\right)$ with the $A_{i}$ finitely generated over $\mathbb{Z}$.

Recall also that if $\widetilde{X}$ is a $k$-scheme, $k$ a field, a point $x \in \widetilde{X}$ is $k$-rational if the natural morphism

$$
k \hookrightarrow k(x)
$$

is an isomorphism, with $k(x)$ the residue field of $x$. (Note at this point that a homomorphism of fields $f \longrightarrow g$ is necessarily injective.) A morphism

$$
\operatorname{Spec}(L) \longrightarrow \widetilde{X}
$$

with $L / k$ a field extension, is completely determined by the choice of a point $x \in$ $\widetilde{X}$ (namely the image of $\operatorname{Spec}(L)$ in $\widetilde{X}$ ) and a field extension $L / k(x)$. Whence the set of $L$-rational points of $\widetilde{X}$ can be identified with

$$
\operatorname{Hom}_{k}(\operatorname{Spec}(L), \widetilde{X}) .
$$

(If $\widetilde{X} \cong \operatorname{Spec}(A)$ is affine, $A$ being a commutative ring, one also has the identification with $\operatorname{Hom}(A, L)$.)

In the next proposition, a $k$-scheme $X \mapsto \operatorname{Spec}(k)$ is said to be locally of finite type (over $k$ ) if $X$ has a cover of open affine subschemes $\operatorname{Spec}\left(A_{i}\right)$, with all the $A_{i}$ finitely generated $k$-algebras. 
Proposition 6.1 (Closed and rational points). (1) Let $X$ be a $\mathbb{Z}$-scheme of finite type. A point $x$ of $X$ is closed if and only if its residue field $k(x)$ is finite.

(2) Let $k=\bar{k}$ be algebraically closed, and let $\widetilde{X} \longrightarrow \operatorname{Spec}(k)$ be a $k$-scheme which is locally of finite type. Then a point $x$ is closed if and only if it is $k$-rational.

(3) More generally, let $k$ be any field. Then a point $x$ of the $k$-scheme $\widetilde{X} \longrightarrow$ $\operatorname{Spec}(k)$, which is again assumed to be locally of finite type, is closed if and only if the field extension $k(x) / k$ is finite. A closed point is $k$-rational if and only if $k(x)=k$.

Assume again that $X$ is an arithmetic scheme. Let $\bar{X}$ be the "atomization" of $X$; it is the set of closed points, equipped with the discrete topology and the sheaf of fields $\{k(x) \mid x\}$. For $x \in \bar{X}$, let $N(x)$ be the cardinality of the finite field $k(x)$, that is, the norm of $x$. Define the arithmetic zeta function $\zeta_{X}(s)$ as

$$
\zeta_{X}(s):=\prod_{x \in \bar{X}} \frac{1}{1-N(x)^{-s}} .
$$

\section{Examples}

We mention four standard examples.

Dedekind. Let $X=\operatorname{Spec}(A)$, where $A$ is the ring of integers of a number field $\mathbb{K}$; then $\zeta_{X}(s)$ is the Dedekind zeta function of $\mathbb{K}$.

Riemann. Put $X=\operatorname{Spec}(\mathbb{Z})$; then $\zeta_{X}(s)$ becomes the classical Riemann zeta function.

Affine space. With $\mathbb{A}^{n}(X)$ being the affine $n$-space over a scheme $X, n \in \mathbb{N}$, one has

$$
\zeta_{\mathbb{A}^{n}(X)}=\zeta_{X}(s-n) .
$$

Projective space. With $\mathbb{P}^{n}(X)$ being the projective $n$-space over a scheme $X$, $n \in \mathbb{N}$, one has

$$
\zeta_{\mathbb{P} n}(X)=\prod_{j=0}^{n} \zeta_{X}(s-j) .
$$

The latter can be obtained inductively by using the expression for the zeta function of affine spaces. 


\subsection{Kurokawa theory}

In [14], Kurokawa says a scheme $X$ is of $\mathbb{F}_{1}$-type if its arithmetic zeta function $\zeta_{X}(s)$ can be expressed in the form

$$
\zeta_{X}(s)=\prod_{k=0}^{n} \zeta(s-k)^{a_{k}}
$$

with the $a_{k} \mathrm{~s}$ in $\mathbb{Z}$. A very interesting result in [14] reads as follows:

Theorem 6.2 (Kurokawa [14]). Let $X$ be a $\mathbb{Z}$-scheme. The following are equivalent.

(i)

$$
\zeta_{X}(s)=\prod_{k=0}^{n} \zeta(s-k)^{a_{k}}
$$

with the $a_{k} s$ in $\mathbb{Z}$.

(ii) For all primes $p$ we have

$$
\zeta_{X \mid \mathbb{F}_{p}}(s)=\prod_{k=0}^{n}\left(1-p^{k-s}\right)^{-a_{k}}
$$

with the $a_{k} s$ in $\mathbb{Z}$.

(iii) There exists a polynomial $N_{X}(Y)=\sum_{k=0}^{n} a_{k} Y^{k}$ such that

$$
\# X\left(\mathbb{F}_{p^{m}}\right)=N_{X}\left(p^{m}\right)
$$

for all finite fields $\mathbb{F}_{p^{m}}$.

Kurokawa defines the $\mathbb{F}_{1}$-zeta function of a $\mathbb{Z}$-scheme $X$ which is defined over $\mathbb{F}_{1}$ as

$$
\zeta_{X \mid \mathbb{F}_{1}}(s):=\prod_{k=0}^{n}(s-k)^{-a_{k}}
$$

with the $a_{k}$ s as above. Define, again as above, the Euler characteristic

$$
\# X\left(\mathbb{F}_{1}\right):=\sum_{k=0}^{n} a_{k} .
$$

The connection between $\mathbb{F}_{1}$-zeta functions and arithmetic zeta functions is explained in the following theorem, taken from [14]. 
Theorem 6.3 (Kurokawa [14]). Let $X$ be a $\mathbb{Z}$-scheme which is defined over $\mathbb{F}_{1}$. Then

$$
\zeta_{X \mid \mathbb{F}_{1}}(s)=\lim _{p \longrightarrow 1} \zeta_{X \mid \mathbb{F}_{p}}(s)(p-1)^{\# X\left(\mathbb{F}_{1}\right)} .
$$

Here, $p$ is seen as a complex variable (so that the left hand term is the leading coefficient of the Laurent expansion of $\zeta_{X \mid \mathbb{F}_{1}}(s)$ around $\left.p=1\right)$.

Theorem 6.3 was also obtained by Soulé in [18].

\section{Examples}

For affine and projective spaces, we obtain the following zeta functions (over $\mathbb{Z}$, $\mathbb{F}_{p}$ and $\mathbb{F}_{1}$, with $n \in \mathbb{N}^{\times}$).

Affine space. $\quad \zeta_{\mathbb{A}^{n} \mid \mathbb{Z}}(s)=\zeta(s-n)$;

$$
\begin{aligned}
& \zeta_{\mathbb{A}^{n} \mid \mathbb{F}_{p}}(s)=\left(1-p^{n-s}\right)^{-1} \\
& \zeta_{\mathbb{A}^{n} \mid \mathbb{F}_{1}}(s)=(s-n)^{-1} .
\end{aligned}
$$

Projective space. $\quad \zeta_{\mathbb{P} n} \mid \mathbb{Z}(s)=\zeta(s) \zeta(s-1) \cdots \zeta(s-n)$;

$$
\begin{aligned}
\zeta_{\mathbb{P} n} \mid \mathbb{F}_{p} & (s)=\left(\left(1-p^{-s}\right)\left(1-p^{1-s}\right) \cdots\left(1-p^{n-s}\right)\right)^{-1} ; \\
\zeta_{\mathbb{P}^{n} \mid \mathbb{F}_{1}}(s) & =(s(s-1) \cdots(s-n))^{-1} .
\end{aligned}
$$

\section{Graphs and zeta functions}

In this section we will introduce a new zeta function for (loose) graphs through $\mathbb{F}_{1}$-theory, following the work of [16].

In [19], starting with a loose graph $\Gamma$, which is a graph in which one also allows edges with 0 or 1 point, I associated a Deitmar scheme $S(\Gamma)$ to $\Gamma$ of which the closed points correspond to the vertices of $\Gamma$. (So by definition, a loose graph is an incidence structure $\Gamma=(V, E)$ with $V$ a set of vertices and $E$ a set of edges, such that each edge is incident with at most 2 vertices.)

Some features of $\mathrm{S}(\cdot)$ :

- Fundamental properties and invariants of the Deitmar scheme can be obtained from the combinatorics of the loose graph, such as connectedness and the isomorphism class of the automorphism group.

- A number of combinatorial $\mathbb{F}_{1}$-objects (such as combinatorial $\mathbb{F}_{1}$-projective space) are just loose graphs, and moreover, the associated Deitmar 
schemes are precisely the "scheme versions" in Deitmar's theory of these objects. (Example: starting from a complete graph with $m+1$ vertices, one obtains the Deitmar scheme of projective space of dimension $m$; this is the "scheme version" of the corresponding combinatorial projective space.)

Translation properties such as in the first item above, were a main goal of the note [19]: trying to handle $\mathbb{F}_{1}$-scheme theoretic issues at the graph theoretic level (bearing in mind how some standard loose graphs should give rise to some standard Deitmar schemes). After base extension, some basic properties of the "real" schemes might then be controlled by the loose graphs, etc.

The idea of the recent work [16] is now to associate a Deitmar constructible set to a loose graph in a more natural way, and to show that, after having applied Deitmar's $\left(\cdot \otimes_{\mathbb{F}_{1}} \mathbb{Z}\right)$-functor, the obtained Grothendieck schemes are defined over $\mathbb{F}_{1}$ in Kurokawa's sense. So they come with a Kurokawa zeta function, and that is the zeta function we associate to loose graphs.

As in [16], we will call the modified functor "F." It has to obey a tight set of rules, of which we mention a few important ones:

Rule \#1 The loose graphs of the affine and projective space Deitmar schemes should correspond to affine and projective space Deitmar schemes.

Rule \#2 A vertex of degree $m$ should correspond locally to an affine space $\mathbb{A}^{m}$.

Rule \#3 An edge without vertices should correspond to a multiplicative group.

Rule \#4 "The loose graph is the map to gluing."

Remark 7.1. - Because of Rule \#1, the pictures of Tits and KapranovSmirnov of affine and projective spaces over $\mathbb{F}_{1}$ are in agreement with the functor $\mathrm{F}$. (This was also the case for the functor S.)

- In general, Rule \#2 does not hold for the functor S. As we expressed at the end of the first section (in the discussion about the functor $\mathcal{A}$ ), this property is highly desirable though.

- Rule \#3 implies that we have to work with a more general version of Deitmar schemes, since we allow expressions of type

$$
\mathbb{F}_{1}[X, Y] /(X Y=1)
$$

(where the last equation generates a congruence on $\mathbb{F}_{1}[X, Y]$ ). In [19], I only worked with Deitmar schemes, thus yielding a less natural approach to what the effect on deleting edges is on the corresponding schemes. By the way, $\mathbb{F}_{1}[X, Y]$ denotes the free abelian monoid generated multiplicatively by $X$ and $Y$, enriched with a zero. 
- The last rule means that for any two vertices $u, v$ of a loose graph $\Gamma$, the intersection of the local affine spaces $\mathbb{A}_{u}$ and $\mathbb{A}_{v}$ which arise in $\mathrm{F}(\Gamma)$ as defined by Rule $\# 2$, can be read from $\Gamma$. In general, this is a highly nontrivial game to play, as the examples and booby traps in [16] show.

For the details, we refer the reader to [16].

\subsection{The Grothendieck ring over $\mathbb{F}_{1}$}

Many of the formulas and calculations in [16] are expressed in the language of Grothendieck rings.

Definition 7.2. The Grothendieck ring of (Deitmar) schemes of finite type over $\mathbb{F}_{1}$, denoted as $K_{0}\left(\mathrm{Sch}_{\mathbb{F}_{1}}\right)$, is generated by the isomorphism classes of schemes $X$ of finite type over $\mathbb{F}_{1},[X]_{\mathbb{F}_{1}}$, with the relation

$$
[X]_{\mathbb{F}_{1}}=[X \backslash Y]_{\mathbb{F}_{1}}+[Y]_{\mathbb{F}_{1}}
$$

for any closed subscheme $Y$ of $X$, and with the product structure given by

$$
[X]_{\mathbb{F}_{1}} \cdot[Y]_{\mathbb{F}_{1}}=\left[X \times_{\mathbb{F}_{1}} Y\right]_{\mathbb{F}_{1}} .
$$

Denote by $\underline{\mathbb{L}}=\left[\mathbb{A}_{\mathbb{F}_{1}}^{1}\right]_{\mathbb{F}_{1}}$ the class of the affine line over $\mathbb{F}_{1}$. Then the multiplicative group $\mathbb{G}_{m}$ satisfies

$$
\left[\mathbb{G}_{m}\right]_{\mathbb{F}_{1}}=\underline{\mathbb{L}}-1,
$$

since it can be identified with the affine line minus one point.

If $X$ is a Deitmar scheme of finite type, and

$$
[X]_{\mathbb{F}_{1}} \in \mathbb{Z}[\underline{\mathbb{L}}] \subset K_{0}\left(\operatorname{Sch}_{\mathbb{F}_{1}}\right),
$$

then we say that $[X]_{\mathbb{F}_{1}}=: \mathrm{P}(X)$ is the Grothendieck polynomial of $X$. More generally, if $\Gamma$ is a loose graph, and there is a polynomial $\mathrm{P}(Z) \in \mathbb{Z}[Z]$ such that for all finite fields $\mathbb{F}_{q}$ we have that

$$
\mathrm{P}(q)=\left|\mathrm{F}(\Gamma) \otimes_{\mathbb{F}_{1}} \mathbb{F}_{q}\right|_{q},
$$

then we say that $\mathrm{P}(Z)=: \mathrm{P}(\Gamma)$ is a Grothendieck polynomial for $\Gamma$ (or $\mathrm{F}(\Gamma)$, or $\left.\mathrm{F}(\Gamma) \otimes_{\mathbb{F}_{1}} \mathbb{F}_{q}\right)$. 


\subsection{Affection principle}

Starting from a (finite) loose graph $\Gamma$, we denote the Deitmar constructible set obtained by applying the functor $\mathrm{F}$ by $\mathrm{F}(\Gamma)$, as before.

In [16] it is shown that each $F(\Gamma)$ comes with a Grothendieck polynomial. Let $\mathrm{P}(\Gamma)$ be the Grothendieck polynomial of $\mathrm{F}(\Gamma)$. For each finite field $\mathbb{F}_{q}$, the number of $\mathbb{F}_{q}$-rational points of $\mathrm{F}(\Gamma) \otimes_{\mathbb{F}_{1}} \mathbb{F}_{q}$ is given by substituting the value $q$ for the indeterminate $\underline{\mathbb{L}}$ in $\mathrm{P}(\Gamma)$ [16]. By Rule \#3, locally each closed point of $\mathrm{F}(\Gamma) \otimes_{\mathbb{F}_{1}} \mathbb{F}_{q}$ yields an affine space (of which the dimension is the degree of the point in the graph), so the total number of points can be expressed through the Inclusion-Exclusion principle.

Consider a finite loose graph $\Gamma$, and let $\mathrm{P}(\Gamma)$ be as above. Taking any edge $u v$ which is not loose, we want to compare $\mathrm{P}(\Gamma)$ and $\mathrm{P}\left(\Gamma_{u v}\right)$ in order to introduce a recursive procedure to simplify the loose graph (in that the number of cycles is reduced). Here, $\Gamma_{u v}$ is the loose graph which one obtains when deleting the edge $u v$, while replacing it by two new loose edges, one through $u$ and one through $v$.

In this section, $\overline{\mathbb{A}}$ denotes the projective completion of a given affine space $\mathbb{A}$. Also, if $\Gamma$ is a loose graph, $\mathbb{P}(\Gamma)$ is the projective $\mathbb{F}_{1}$-space which is defined on the ambient graph of $\Gamma$ (i.e., the smallest graph in which $\Gamma$ is embedded).

Calling $\mathrm{d}(\cdot, \cdot)$ the distance function in $\Gamma$ defined on $V \times V, V$ being the vertex set (so that, for example, $\mathrm{d}(s, t)$, with $s$ and $t$ distinct vertices, is the number of edges in a shortest path from $s$ to $t$ ), it appears that one only needs to consider what happens in the vertex set

$$
\mathbf{B}(u, 1) \cup \mathbf{B}(v, 1),
$$

where $\mathbf{B}(c, k):=\{v \in V \mid \mathrm{d}(c, v) \leq k\}$.

Theorem 7.3 (Affection Principle [16]). Let $\Gamma$ be a finite connected loose graph, let $x y$ be an edge on the vertices $x$ and $y$, and let $S$ be a subset of the vertex set. Let $k$ be any finite field, and consider the $k$-constructible set $\mathrm{F}(\Gamma) \otimes_{\mathbb{F}_{1}} k$. Then $\cap_{s \in S} \mathbb{A}_{s}$ changes when one resolves the edge xy only if $|S \cap(\mathbf{B}(x, 1) \cup \mathbf{B}(y, 1))| \geq 2$.

In the next theorem, we will use the notation $\mathbb{P}(\mathbf{B}(u, 1) \cup \mathbf{B}(v, 1))=: \mathbb{P}_{u, v}$. If $\Delta$ is a loose graph, its reduced version is the graph one obtains after deleting the loose edges.

Corollary 7.4 (Geometrical Affection Principle [16]). Let $\Gamma$ be a finite connected loose graph, let $x y$ be an edge on the vertices $x$ and $y$, and let $k$ be any finite field. The difference in the number of k-points of $\mathrm{F}(\Gamma) \otimes_{\mathbb{F}_{1}} k$ and $\mathrm{F}\left(\Gamma_{x y}\right) \otimes_{\mathbb{F}_{1}} k$ is

$$
\left|\mathrm{F}\left(\Gamma_{\mid \mathbb{P}_{x, y}}\right) \otimes_{\mathbb{F}_{1}} k\right|_{k}-\left|\mathrm{F}\left(\Gamma_{x y \mid \mathbb{P}_{x, y}}\right) \otimes_{\mathbb{F}_{1}} k\right|_{k} .
$$


In this expression, $\Gamma$ may be chosen to be reduced (but one is not allowed to reduce $\left.\Gamma_{x y}\right)$.

In terms of Grothendieck polynomials, we have the following theorem.

Corollary 7.5 (Polynomial Affection principle [16]). Let $\Gamma$ be a finite connected loose graph, let $x y$ be an edge on the vertices $x$ and $y$, and let $k$ be any finite field. Then we have

$$
\mathrm{P}(\Gamma)-\mathrm{P}\left(\Gamma_{x y}\right)=\mathrm{P}\left(\Gamma_{\mid \mathbb{P}_{x, y}}\right)-\mathrm{P}\left(\Gamma_{x y \mid \mathbb{P}_{x, y}}\right) .
$$

\subsection{Loose trees}

A loose tree is a loose graph without cycles.

Let $\Gamma$ be a loose tree.

- Let $D$ be the set of degrees $\left\{d_{1}, \ldots, d_{k}\right\}$ of the vertex set $V(\Gamma)$ such that $1<d_{1}<d_{2}<\ldots<d_{k}$.

- Let us call $n_{i}$ the number of vertices of $\Gamma$ of degree $d_{i}, 1 \leq i \leq k$.

- Put $I=\sum_{i=1}^{k} n_{i}-1$.

- Let $E$ be the number of vertices of $\Gamma$ with degree 1, that is the end points.

Then by [16], the class of $\Gamma$ in $K_{0}\left(\mathrm{Sch}_{\mathbb{F}_{1}}\right)$ is given by

$$
[\Gamma]_{\mathbb{F}_{1}}=\sum_{i=1}^{k} n_{i} \underline{\underline{L}}^{d_{i}}-I \cdot \underline{\mathbb{L}}+I+E .
$$

\subsection{Surgery}

Calculating Grothendieck polynomials of general loose graphs is very complicated - see the many examples analyzed in [16]. In loc. cit., a procedure called "surgery" is introduced, which makes it possible to determine such polynomials by "resolution of edges," eventually reducing the calculation to the tree case, and this is a case which was resolved completely (cf. $\S 7.3$ ).

When $\Delta$ is a loose graph, and $e=x y$ is an edge with vertices $x$ and $y \neq x$, resolving $e$ means that one constructs the loose graph $\Delta_{x y}=\Delta_{e}$ as before, i.e., the adjacency between $x$ and $y$ is broken, and replaced by two new loose edges (one on $x$ and one on $y$ ). (Locally, the dimensions of the affine spaces at $x$ 
and $y$ remain the same, and the dimension of the ambient projective space of $\Delta$ increases.)

In a nutshell, the following happens, starting from a finite loose graph $\Gamma=$ $(V, E)$.

Spanning. Choose an arbitrary loose spanning tree $T$ (obviously defined) in $\Gamma$.

Resolution. Let $S$ be the set of egdes of $\Gamma$ not in $T$ which are not loose. Order $S=\left\{e_{1}, \ldots, e_{n}\right\}$. Now resolve all the edges in $S$, as follows:

$$
\Gamma \longrightarrow \Gamma_{e_{1}} \longrightarrow\left(\Gamma_{e_{1}}\right)_{e_{2}} \longrightarrow \cdots
$$

while keeping track of all the polynomial differences

$$
\left[\mathrm{P}\left(\Gamma_{e_{1}}\right)-\mathrm{P}(\Gamma)\right], \quad\left[\mathrm{P}\left(\left(\Gamma_{e_{1}}\right)_{e_{2}}\right)-\mathrm{P}\left(\Gamma_{e_{1}}\right)\right], \ldots
$$

which one calculates using the Affection Principle.

Reduction. Once one has resolved all the edges in $S$, we obtain a tree, and by $\S 7.3$ we know its Grothendieck polynomial. Now use the list of differences in the previous step to write down the Grothendieck polynomial of $\Gamma$.

In [16] it is shown that surgery is independent of the choice of the spanning tree, and of the order in which one chooses to resolve the edges.

\subsection{Resolving edges - two examples}

We now explain some examples.

\subsubsection{Example \# 1}

We define $\Gamma(u, v ; 2)$, with $u, v$ symbols, to be the loose graph with adjacent vertices $u, v ; 2$ common neighbors $v_{1}, v_{2}$ of $u$ and $v$ and no further incidences.

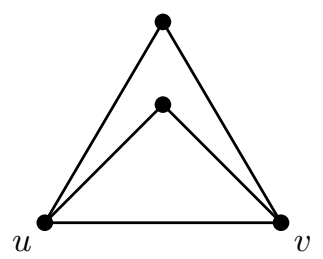


For any field $k$, the corresponding $k$-constructible sets consist of two affine 3 -spaces $\mathbb{A}_{u}$ and $\mathbb{A}_{v}$ and 2 additional closed points in their spaces at infinity, of which the union covers all the points of the projective 3 -space $\mathbb{P}(\Gamma(u, v ; 2))$ up to all points of the intersection $\gamma$ of their spaces at infinity (which is a projective line), except 2 points in $\gamma$ in general position. So the Grothendieck polynomial is

$$
\sum_{i=0}^{3} \underline{\mathbb{L}}^{i}-\left(\left(\sum_{i=0}^{1} \underline{\mathbb{L}}^{i}\right)-2\right)=\underline{\mathbb{L}}^{3}+\underline{\mathbb{L}}^{2}+2 .
$$

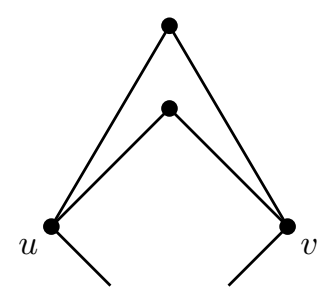

Resolving $\Gamma(u, v ; 2)$ along $u v$, the $k$-constructible sets corresponding to $\Gamma(u, v ; 2)_{u v}$ consist of two disjoint affine 3 -spaces $\mathbb{A}_{u}$ and $\mathbb{A}_{v}$ (of which the planes at infinity intersect in the projective line generated by $v_{1}, v_{2}$ ) and 2 additional mutually disjoint affine planes $\alpha_{i}, i=1,2$, in the projective 5 -space $\mathbb{P}(\Gamma(u, v ; 2))$ such that for each $j, \alpha_{j} \cap \mathbb{A}_{u} \cong \alpha_{j} \cap \mathbb{A}_{v}$ is a projective line minus two points.

The Grothendieck polynomial is

$$
2 \underline{\mathbb{L}}^{3}+2 \underline{\mathbb{L}}^{2}-4(\underline{\mathbb{L}}-1) .
$$

\subsubsection{Example \#2}

Starting from a triangle (as a graph), i.e., a combinatorial projective plane over $\mathbb{F}_{1}$, one deduces in a similar manner that its Grothendieck polynomial is $\underline{\underline{L}}^{2}+$ $\underline{\mathbb{L}}+1$. In general, the Grothendieck polynomial of a complete graph with $m+1$ vertices, $m \neq 0$, is

$$
\underline{\mathbb{L}}^{m}+\underline{\mathbb{L}}^{m-1}+\cdots+1 .
$$

The loose graph of an affine $\mathbb{F}_{1}$-space of dimension $m$ has as Grothendieck polynomial

$$
\underline{\mathbb{L}}^{m} \text {. }
$$

Both (63) and (64) are connected via the expression (15) in the Grothendieck ring. 


\subsection{The zeta function}

We formally recall the next theorem (which was already mentioned implicitly), from [16].

Theorem 7.6 ([16]). For any loose graph $\Gamma$, the $\mathbb{Z}$-constructible set $\chi:=F(\Gamma) \otimes_{\mathbb{F}_{1}}$ $\mathbb{Z}$ is defined over $\mathbb{F}_{1}$ in Kurokawa's sense.

Theorem 7.6 makes it possible to associate a (Kurokawa) zeta function to any loose graph, in the following way.

Definition 7.7 (Zeta function for (loose) graphs). Let $\Gamma$ be a loose graph, and let $\chi:=\mathrm{F}(\Gamma) \otimes_{\mathbb{F}_{1}} \mathbb{Z}$. Let $\mathrm{P}(\Gamma)=\sum_{i=0}^{m} a_{i} X^{i} \in \mathbb{Z}[X]$ be as above. We define the $\mathbb{F}_{1}$-zeta function of $\Gamma$ as:

$$
\zeta_{\Gamma}^{\mathbb{F}_{1}}(t):=\prod_{k=0}^{m}(t-k)^{-a_{k}}
$$

\subsection{Example: trees}

Now let $\Gamma$ be a tree. We use the same notation as before, so that its class in the Grothendieck ring is given by

$$
[\Gamma]_{\mathbb{F}_{1}}=\sum_{i=1}^{m} n_{i} \underline{\underline{L}}^{d_{i}}-I \cdot \underline{\mathbb{L}}+I+E .
$$

The zeta function is thus given by

$$
\zeta_{\Gamma}^{\mathbb{F}_{1}}(t)=\frac{(t-1)^{I}}{t^{E+I}} \cdot \prod_{k=1}^{m}(t-k)^{-n_{k}} .
$$




\section{A Acquiring flesh (2) - The Weyl functor depicted}

Sometimes, the functor $\mathcal{A}$ is artfully depicted by the following diagram, in which Bacon's "Study after Velázquez's portrait of Pope Innocent X" [1] is compared to Velázquez's "Portrait of Innocent X" [29] (Bacon's version being the $\mathbb{F}_{1}$-version of the original painting of Velázquez):

\section{"Portrait of Innocent X"}

An oil on canvas $(114 \mathrm{~cm} \times 119 \mathrm{~cm})$ of the Spanish painter Diego Velázquez (1599-1660) dating from about 1650, depicting a portrait of Pope Innocent X.

\section{$\downarrow \mathcal{A}$}

"Study after Velázquez's portrait of Pope Innocent X"

An oil on canvas $(153 \mathrm{~cm} \times 118 \mathrm{~cm})$ of the Irish painter Francis Bacon (1909-1992) dating from 1953, showing a distorted version of Velázquez's portrait of Pope Innocent X.

At the conference, I showed that in a more modern setting, there is some analogy with the arrow

$$
\mathrm{JAT} \longrightarrow \mathrm{KT} \text {. }
$$




\section{References}

[1] F. Bacon, Study after Velázquez's portrait of Pope Innocent X, Wikipedia page: https://en.wikipedia.org/wiki/Study_after_Velázquez's_Portrait_of_Pope_Innocent_X.

[2] F. Buekenhout and E. Shult, On the foundations of polar geometry, Geom. Dedicata 3 (1974), 155-170.

[3] F. Buekenhout and A. Sprague, Polar spaces having some line of cardinality two, J. Combin. Theory, Ser. A 33 (1982), 223-228.

[4] H. Cohn, Projective geometry over $\mathbb{F}_{1}$ and the Gaussian binomial coefficients, Amer. Math. Monthly 111 (2004), 487-495.

[5] A. Deitmar, Schemes over $\mathbb{F}_{1}$, in Number Fields and Function Fields - Two Parallel Worlds, Progr. Math. 239, 2005, Birkhäuser Boston, Inc., Boston, MA, pp. 87-100.

[6] , Remarks on zeta functions and $K$-theory over $\mathbb{F}_{1}$, Proc. Japan Acad. Ser. A Math. Sci. 82 (2006), 141-146.

[7], $\mathbb{F}_{1}$-Schemes and toric varieties, Beiträge Algebra Geom. 49 (2008), 517-525.

[8] C. Deninger, On the $\Gamma$-factors attached to motives, Invent. Math. 104 (1991), 245-261.

[9] , Local $L$-factors of motives and regularized determinants, Invent. Math. 107 (1992), 135-150.

[10] , Motivic $L$-functions and regularized determinants, in Motives (Seattle, WA, 1991), Proc. Sympos. Pure Math. 55, 1994, pp. 707-743.

[11] S. Haran, Index theory, potential theory, and the Riemann hypothesis, in L-functions and arithmetic (Durham, 1989), pp. 257-270, London Math. Soc. Lecture Note Ser. 153, Cambridge Univ. Press, Cambridge, 1991.

[12] M. Kapranov and A. Smirnov, Cohomology determinants and reciprocity laws: number field case, Unpublished preprint.

[13] K. Kato, Toric singularities, Amer. J. Math. 116 (1994), 1073-1099.

[14] N. Kurokawa, Zeta functions over $\mathbf{F}_{1}$, Proc. Japan Acad. Ser. A Math. Sci. 81 (2005), 180-184. 
[15] Yu. Manin, Lectures on zeta functions and motives (according to Deninger and Kurokawa), Columbia University Number Theory Seminar (New York, 1992), Astérisque 228 (1995), 121-163.

[16] M. Mérida-Angulo and K. Thas, Deitmar schemes, graphs and zeta functions, J. Geom. Physics 117 (2017), 234-266.

[17] A. L. Smirnov, Hurwitz inequalities for number fields, (Russian) Algebra $i$ Analiz 4 (1992), 186-209. Translation in St. Petersburg Math. J. 4 (1993), 357-375.

[18] C. Soulé, Les variétés sur le corps à un élément, Mosc. Math. J. 4 (2004), 217-244, 312.

[19] K. Thas, The structure of Deitmar schemes, I, Proc. Japan Acad. Ser. A Math. Sci. 90 (2014), 21-26.

[20] _ The Weyl functor — Introduction to Absolute Arithmetic, in Absolute Arithmetic and $\mathbb{F}_{1}$-Geometry (K. Thas, ed.), EMS Publishing House, Zürich, pp. 3-38, 2016.

[21] Arithmetic and $\mathbb{F}_{1}$-Geometry (K. Thas, ed.), EMS Publishing House, Zürich, pp. 83-159, 2016.

[22] _ A taste of Weil theory in characteristic one, in Absolute Arithmetic and $\mathbb{F}_{1}$-Geometry (K. Thas, ed.), EMS Publishing House, Zürich, pp. 365386, 2016.

[23] — (ed.) Absolute Arithmetic and $\mathbb{F}_{1}$-Geometry, EMS Publishing House, Zürich, 2016.

[24] _ Projective spaces over $\mathbb{F}_{1^{\ell}}$, Preprint, 18pp., 2016.

[25] Unpublished notes.

[26] J. Tits, Sur les analogues algébriques des groupes semi-simples complexes, Centre Belge Rech. Math., Colloque d'Algèbre supérieure, Bruxelles du 19 au 22 déc. 1956, pp. 261-289, 1957.

[27] H. Van Maldeghem, Generalized Polygons, Monographs in Math. 93, Birkhäuser Verlag, Basel, 1998.

[28] O. Veblen and J. W. Young, A set of assumptions for projective geometry, Amer. J. Math. 30 (1908), 347-380. 
[29] D. Velázquez, Portrait of Innocent X, Wikipedia page: https://en.wikipedia.org/wiki/Portrait_of_Innocent_X.

Koen Thas

Department of Mathematics, Ghent University, KriJgslaAn 281, S25, B-9000 GHent, BeLGIUM

e-mail: koen.thas@gmail.com 